\title{
Estratégias de analgesia multimodal no manejo da dor aguda em adultos na emergência
}

\author{
Multimodal analgesia strategies in the management of acute pain in adults in the \\ emergency room
}

Estrategias de analgesia multimodal en el manejo del dolor agudo en adultos en urgencias

Marina Sammarco Eziliano ${ }^{* *}$, Aline Dias da Silva², Amanda Marinho Lourenço², Bernardo Valle Zanetti $^{3}$, Herton Algenir Garcia dos Santos Júnior ${ }^{4}$, Isabella Barboza Mendonça ${ }^{5}$, Liz Ulm Ferreira Velloso ${ }^{4}$, Paula Ariane Toneli Reis ${ }^{6}$, Vitória Carolina Bitencourt da Silva ${ }^{7}$, Thales Miranda Sales ${ }^{8}$.

\section{RESUMO}

Objetivo: A dor é uma das principais queixas do pronto atendimento e torna-se um desafio por ser subjetiva e individual, infligida ou não por uma lesão tecidual. Assim, destaca-se a importância de descrever 0 tratamento multimodal do paciente adulto com dor aguda na emergência. Revisão bibliográfica: $O$ manejo na terapia multimodal visa a combinação de diferentes classes farmacológicas, ou tratamentos não farmacológicos, esses não muito viáveis no atendimento emergencial. Quanto aos fármacos utilizados temos os anestésicos locais, AINEs e opioides que são os tradicionais, enquanto os anticonvulsivantes, agonistas alfa 2 adrenérgicos e os NMDA são os não tradicionais. Os medicamentos não tradicionais são utilizados como adjuvantes aos opioides ou potenciais substitutos, que visam justamente a melhor adequação da analgesia do paciente diminuindo as doses de opioides e seus efeitos colaterais. No entanto, o tratamento médico de emergência na analgesia é um ponto conflitante, quanto a sua dosagem e efeitos adversos. Considerações finais: $O$ tratamento da dor é sempre individualizado, mas em uma perspectiva ampla as terapias multimodais são boas escolhas no tratamento da dor aguda, principalmente na diminuição da utilização de opioides. Entretanto, ainda é necessário um número maior de estudos para que o manejo seja melhor compreendido.

Palavras-chave: Dor, Analgesia, Emergências.

\begin{abstract}
Objective: Pain is one of the main complaints in the emergency department (ED) and becomes a challenge because it is specific and individual, inflicted or not by a tissue injury. Thus, the importance of describing the multimodal treatment of adult patients with acute pain in the emergency is highlighted. Bibliographic review: Management in multimodal therapy aims at combining different pharmacological classes, or nonpharmacological treatments, which are not very viable in ED. As for the drugs used, there are the local anesthetics, Non-Steroidal Anti Inflammatory Drugs (NSAID) and opioids that are the traditional ones, while the anticonvulsants, alpha 2 adrenergic agonists and N-methyl-D-aspartate (NMDA) are the non-traditional ones. Non-traditional medications are used as adjuvants to opioids or potential substitutes, which aim precisely at adapting the patient's analgesia by decreasing opioid doses and its side effects. However, treatment for analgesia in ED is a conflicting point in terms of its dosage and adverse effects. Final considerations: That pain treatment is always individualized, but in a broad perspective as multimodal therapies are good choices in the treatment of acute pain, mainly in reducing the use of opioids. However, a greater number of studies is still necessary for the management to be better understood.
\end{abstract}

Key words: Pain, Analgesia, Emergencies.

${ }^{1}$ Universidade Federal de Minas Gerais (UFMG), Belo Horizonte - MG. *E-mail: m.eziliano@gmail.com

2 Universidade José do Rosário Vellano (Unifenas), Belo Horizonte - MG.

${ }^{3}$ Faculdade de Ciências Médicas e da Saúde de Juiz de Fora (SUPREMA), Juiz de Fora - MG.

${ }_{4}^{4}$ Universidade Salvador (UNIFACS), Salvador - BA.

5 Universidade Vila Velha (UVV), Vila Velha - ES.

6 Instituto Master de Ensino Presidente Antônio Carlos (IMEPAC), Araguari - MG.

${ }^{7}$ Centro Universitário de Várzea Grande (UNIVAG), Várzea Grande - MT.

${ }^{8}$ Centro Universitário de Caratinga (UNEC), Caratinga - MG. 


\section{RESUMEN}

Objetivo: El dolor es una de las principales quejas de la atención de emergencia y se convierte en un desafío porque es subjetivo y individual, causado o no por lesión tisular. De esta forma, se destaca la importancia de describir el tratamiento multimodal del paciente con dolor agudo en urgencias. Revisión bibliográfica: El manejo en terapia multimodal tiene como objetivo combinar diferentes clases farmacológicas, o tratamientos no farmacológicos, que son poco viables en la atención de urgencias. En cuanto a los fármacos utilizados, tenemos los anestésicos locales, AINE y opioides, los tradicionales, mientras que los anticonvulsivos, agonistas adrenérgicos alfa 2 y NMDA son no tradicionales. Los no tradicionales se utilizan como adyuvantes de los opioides o posibles sustitutos, que tienen como objetivo adaptar mejor la analgesia mediante la reducción de las dosis de opioides y sus efectos secundarios. Sin embargo, el tratamiento para la analgesia es un punto conflictivo en cuanto a su dosis y efectos adversos. Consideraciones finales: El tratamiento del dolor siempre es individualizado, pero en una perspectiva amplia, las terapias multimodales son buenas opciones en el tratamiento del dolor agudo, especialmente para disminuir el uso de opioides. Todavía es necesario mayor número de estudios para comprender mejor el manejo.

Palabras clave: Dolor, Analgesia, Urgencias médicas.

\section{INTRODUÇÃO}

Segundo a Sociedade Internacional para o Estudo da Dor, a dor é definida como uma experiência sensorial e emocional desagradável associada a uma lesão tecidual real ou potencial. Decorrente disso, a dor é considerada como um dos principais motivos de martírio da humanidade, provocando incapacidades que repercutem na qualidade de vida, no psicológico e no financeiro. Sendo assim, a dor aguda é uma das principais queixas em serviços de emergência e merece atenção para o diagnóstico e tratamento efetivos, de acordo com os princípios éticos da prática médica (BERTONCELLO K, et al., 2016).

A dor aguda é resultante de uma ativação de nociceptores, mediadores químicos e inflamação, fato esse que contribui para prevenir o dano tecidual. O manejo terapêutico inicial pode ser realizado com Antiinflamatórios Não Esteroidais (AINEs), incluindo os inibidores seletivos da COX2. Observa-se que, para o controle multimodal da dor, em geral, essa classe de medicamentos é utilizada em associação com outras drogas, a fim de evitar possíveis efeitos colaterais e otimizar a analgesia, uma vez que apresenta maior risco de reações indesejadas quando utilizado isoladamente, principalmente em doses maiores, o que pode comprometer a relação custo/benefício, sendo mais eficaz nos casos de dor aguda leve à moderada (FIGUEIREDO W, et al., 2015).

A analgesia multimodal tem por objetivo o alívio da dor por meio de recursos farmacológicos ou não, e recebem esse nome por atuarem de diferentes modos e em diferentes locais no sistema nervoso. Dentre os agentes farmacológicos, se encontram os tradicionais, como os anestésicos locais, os AINES e os opioides, e os não tradicionais compostos pelos anticonvulsivantes, agonistas alfa-2-adrenérgicos, antagonistas do receptor $\mathrm{N}$-metil $\mathrm{D}$-aspartato (NMDA). Em relação às intervenções não farmacológicas, exemplifica-se a estimulação elétrica nervosa transcutânea, a terapia cognitivo-comportamental, entre outras (MANWORREN $\mathrm{R}, 2015)$.

O uso da analgesia multimodal demonstra-se mais eficiente, diminui a utilização de opioides e a redução dos efeitos colaterais, comparado a utilização de modalidade única (CHOU R, et al., 2016). A exposição contínua aos opioides induz a diminuição dos seus efeitos analgésicos, em decorrência disso, os pacientes necessitam de doses cada vez mais elevadas, ampliando assim o risco de dependência, depressão respiratória e overdose. Ademais, os opioides podem ocasionar hiperalgesia, acentuando e não reduzindo a dor (VOLKOW N, et al., 2018).

Estudos dos fenômenos álgicos ganham cada vez mais relevância, uma vez que o manejo adequado do paciente ainda é considerado um desafio frente aos resultados de subtratamento. Avaliações subjetivas são passíveis de erros e resultados de baixa acurácia clínica, fato esse que pode interferir tanto na conduta abordada quanto na qualidade de vida do paciente (BERTONCELLO K, et al., 2016).

Diante do exposto previamente, fica explícita a relevância desse tema na assistência em saúde, portanto, o presente estudo tem como objetivo revisar a bibliografia referente ao tratamento multimodal da dor aguda, 
Devem, ainda, ser consideradas as vias de administração dos fármacos, uma vez que na medicina de emergência os analgésicos necessitam de ação rápida e eficaz. A via intramuscular tem potencial para somar dor localizada, e pode não só ter absorção de maneira indeterminada, bem como seu tempo de ação. Já a via Intravenosa (IV) apesar de requerer acesso ainda é a via mais usual. Além disso, os analgésicos aprovados para serem utilizados por essa via, também podem ser aplicados por meio intraósseo. Por fim, a via intranasal além de possuir uma rápida absorção, é uma estratégia indolor, segura e eficaz. No entanto, a maioria dos analgésicos ainda não tiveram o uso aprovado para serem usados por essa via (HÄSKE D, et al., 2017; SIN B, et al., 2019).

\section{Anestésicos locais}

Os anestésicos locais são usados na forma de infiltração subcutânea e anestesia regional. Há evidências sobre o uso de anestésicos locais de forma sistêmica por via intravenosa, dessa forma não ficando apenas limitados em regiões sob torniquete. Além disso, há relatos de reduções significativas nas necessidades de opioides em pacientes que fizeram uso de lidocaína por via IV durante e após procedimentos cirúrgicos. Assim como benefícios que podem incluir analgesia, anti-hiperalgesia e propriedades anti-inflamatórias por meio de múltiplos mecanismos. No entanto, é necessário cautela com doses em bolus e infusão para evitar toxicidade (BEVERLY A, et al., 2017).

\section{Anti-Inflamatórios Não Esteroidais (AINEs)}

Estudos apontaram que os AINEs são eficazes no controle da dor aguda de intensidade leve a moderada e associada à inflamação ou lesão tecidual. Isso se deve ao fato de reduzirem a produção de prostaglandinas que sensibilizam nociceptores para mediadores da inflamação. No entanto, é relevante destacar que são responsáveis por diversas reações adversas, sendo as principais o prurido, a retenção urinária, a náusea e o vômito, quando utilizados por períodos prolongados, assim como a possibilidade de ocasionar doença renal crônica. Em menor proporção, efeitos adversos como reações cutâneas do tipo urticárias e exantema foram atribuídos especificamente ao uso de ácido acetilsalicílico, paracetamol, ibuprofeno e piroxicam (HAMRICK K, et al., 2019; FIGUEIREDO W, et al., 2015).

Além disso, é válido ressaltar que o efeito analgésico foi observado tanto na utilização isolada quanto na associação com outros fármacos, como pregabalina, alfa-2-agonistas ou cetamina. Ainda, para o controle da dor aguda, o efeito sinérgico entre AINEs e opioides ou anestésicos locais apresenta bom controle álgico e, apesar dos efeitos colaterais, os AINEs apresentam perfil aceitável de segurança (FIGUEIREDO W, et al., 2015).

\section{Sufentanil e outros Opioides}

O alívio da dor aguda em pacientes na emergência pode ser acelerado com terapias usando o sufentanil. Uma estratégia é o uso Intranasal (IN) em dose única de $0,4 \mu \mathrm{g} / \mathrm{kg}$ ainda na triagem do departamento. $\mathrm{O}$ tratamento mais usado para dor aguda é a administração de analgésicos IV, incluindo opioides, após a triagem. Visto isso, a inclusão do sufentanil, na analgesia multimodal usual do pronto-socorro acarreta em um número maior de pacientes com alívio da dor. Pois, a analgesia tem início na triagem, não necessitando de um acesso IV para o começo da administração (LEMOEL F, et al., 2019).

Um dos analgésicos mais usados no departamento de emergência é a morfina por via IV. Quando comparado, o sufentanil por via IN tem efeito semelhante no manejo da dor, considerando um intervalo de até 30 minutos após o início da administração. $\mathrm{Na}$ avaliação da diferença na Escala Numérica de Dor (NRS), nesta lacuna de tempo, há similaridade na eficácia dos fármacos. Porém, ambos podem apresentar efeitos adversos leves como tontura, ondas de calor, náusea/vômito, mau gosto/cheio e reação alérgica leve. Ou ainda, graves como hipoxemia $(\mathrm{SpO} 2<90 \%)$ e hipotensão $(\mathrm{PAS}<90 \mathrm{mmHg})$. Como a limitação da morfina por via IV é o acesso, o sufentanil spray pode trazer eficácia para o manejo da dor no pronto-socorro (BLANCHER $\mathrm{M}$, et al., 2019).

Outra forma de administração para o sufentanil é a sublingual, sendo uma opção válida para manejo da dor aguda moderada a grave no pronto-socorro. Isto por ter início de ação rápido e poder ser administrado na 
triagem do departamento. Dessa forma a analgesia é precoce, podendo o paciente ter alívio da dor mais rápido que o esperado para o departamento (MINER JR, et al., 2017).

\section{Anticonvulsivantes}

Medicamentos utilizados para o tratamento da epilepsia apresentam também propriedades analgésicas devido aos seus mecanismos de ação. Por exemplo, a gabapentina e pregabalina causam redução da liberação de neurotransmissores excitatórios dependente de cálcio, diminuindo assim a excitabilidade neuronal (FINNERUP N, 2019). Alguns estudos apontam que os medicamentos análogos ao ácido gamaaminobutírico (GABA), como a gabapentina, interagem não apenas com os canais de cálcio dependentes de voltagem, mas também com receptores NMDA e com a proteína quinase C (BERVELY A, et al., 2017).

O uso desses medicamentos é bem documentado e inclusive recomendado para o tratamento de dores crônicas, como a Gabapentina para o tratamento da dor neuropática e a pregabalina que apresentou-se eficaz em ensaios para o tratamento da dor da fibromialgia. Além disso, a oxcarbazepina e a carbamazepina são considerados tratamentos de primeira linha para neuralgia do trigêmeo (FINNERUP N, 2019).

O uso perioperatório de gabapentina e pregabalina mostrou um efeito poupador de opioides na dor pósoperatória aguda atuando na analgesia multimodal, no entanto, há uma carência de estudos direcionados ao uso desses medicamentos no controle da dor aguda e nos departamentos de emergência, sendo válido ressaltar que isto pode estar relacionado ao risco aumentado de eventos adversos graves e, portanto, a não recomendação dessa classe como tratamento de rotina para dor. Além disso, há evidências crescentes de uso indevido e abuso dessas drogas (BERVELY A, et al., 2017; FINNERUP N, 2019).

\section{Agonistas alfa-2-adrenérgicos}

Os agonistas alfa-2-adrenérgicos atuam nas vias descendentes da dor, ativando receptores para estimular a liberação de acetilcolina, e nas vias ascendentes da dor, inibindo a liberação da substância $P$ que é um neuropeptídeo ou neurotransmissor liberado do neurônio aferente primário e se liga ao neurônio secundário no corno dorsal para criar um potencial de ação. Assim, a inibição da liberação da substância $P$ reduz a transmissão da dor (MANWORREN R, 2015).

A administração dessa classe de medicamentos em cirurgias, no momento perioperatório e pós-operatório, é bem documentada quanto à eficácia da analgesia e satisfação do paciente. Apesar do uso empírico em emergências, são poucos ainda os estudos que demonstram o controle da dor aguda com essas drogas, necessitando de mais pesquisas não apenas quanto a dosagem de dexmedetomidina, mas também na identificação do efeito dessa droga no sistema imunológico e no mecanismo de ação analgésica (MAO Y, et al., 2020).

\section{Cetamina/antagonista NMDA}

No departamento de emergência (DE), os opioides intravenosos são a base do tratamento para dor aguda. Embora os opioides possam trazer um rápido alívio da dor, as dosagens necessárias para uma adequada analgesia podem trazer efeitos adversos, como sedação excessiva e depressão respiratória. Além disso, a dosagem recomendada de $0,1 \mathrm{mg} / \mathrm{kg}$ para alívio da dor, pode ser ineficaz no controle em quadros agudos graves (BEAUDOIN F, et al., 2014).

Dessa forma, uma alternativa para o controle da dor aguda é a Cetamina. A Cetamina é um antagonista do N-metil D-aspartato (NMDA) e é administrado intramuscular, intranasal, retal, formas subcutâneas e epidurais (MAJIDINEJAD S, et al., 2014). A Cetamina tem sido bastante estudada por ser uma forte candidata a ser uma substituta dos opioides. Assim, o uso de Cetamina em baixas doses tem sido recorrente alvo de estudos quanto à sua aplicação em casos de dor aguda na emergência médica, e seu uso tem sido de manejo singular ou associado (KARLOW N, et al., 2018; BOWER K, et al., 2017).

As Cetaminas possuem efeitos adversos mais elevados quando utilizadas com altas doses, podendo causar: 1) queda na saturação de oxigênio; 2) alucinações vívidas; 3) disforia; 4) agitação. Por isso, seu uso é recomendado em baixas doses. Todavia, suas vantagens quanto as aplicações no paciente são variadas, 
possuindo efeitos como: a) sedação para procedimentos de resgate e procedimentos invasivos; b) proteção contra a dor; c) sedação eficaz em pacientes hemodinamicamente instáveis; d) controla a pressão intracraniana e a pressão pela perfusão cerebral, sendo utilizada em pacientes com traumas cranianos (HÄSKE D, et al., 2017).

Nesse cenário, o uso de Baixa Dose de Cetamina (BDC), doses de 0,1 a 0,6 mg/kg; mais comumente 0,3 $\mathrm{mg} / \mathrm{kg}$, Intravenosa (IV), sem associação a outros medicamentos tem sido uma das principais alternativas aos opioides em quadros de manejo da dor aguda leve à moderada nos Departamentos de emergência, e seus resultados têm se mostrado similares aos obtidos pelos opioides, proporcionando analgesia similar a esses em pacientes adultos (MOTOV S, et al., 2015).

As vantagens do uso da Cetamina como forma de monoterapia são: menor risco de dependência química; melhor efeito terapêutico em pacientes narcóticos; menor complicação respiratória; menor risco de reações adversas que a morfina. Todavia, seu uso também apresenta efeitos colaterais, tais como: a) Laringoespasmo; b) náuseas e vômitos; c) reações emergenciais; d) sintomas de trato urinário baixo (aumento de frequência, urgência, disúria e falha renal). Esse fármaco sofre ainda mecanismo de $1^{\text {a }}$ passagem, necessitando de ajustes na sua dosagem para pacientes com morbidades hepáticas. Assim, a recomendação farmacológica para o manejo da dor aguda vai de acordo com o perfil do paciente, analisando cada caso com os possíveis efeitos adversos dos fármacos, uma vez que sua atuação na analgesia atinge resultados muito similares (KARLOW N, et al., 2018).

Já o uso de Cetaminas em associação com opioides no Departamento de Emergência para o manejo da dor aguda tem apresentado resultados melhores em diversos aspectos quando comparado ao uso monoteraupêutico de opioides, bem como a necessidade de doses menores de opioides quando utilizados em conjunto. A associação entre esses fármacos não apresenta diferenças significativas quanto ao manejo da dor quando comparado ao uso único de opioides, sendo a sua analgesia similar após 30 minutos do manejo inicial, e com valores melhores de redução da dor nos 15 minutos iniciais após a aplicação (BOWER K, et al., 2017; HÄSKE D, et al., 2017; SIN B, et al., 2019).

Todavia, seu ganho terapêutico encontra-se na menor quantidade de doses extras para o controle da dor no paciente, apesar do combinado Cetamina + opioide apresentar maior incidência de efeitos adversos de curta duração, como náusea; tontura; coceira; nistagmo e zumbido (BOWER K, et al., 2017; SIN B, et al., 2019).

Por outro lado, foi visto que quando administrada em bolus único, o efeito analgésico da Cetamina é de curta duração, sendo o efeito de pico nos primeiros minutos após a administração com uma duração de 1015 minutos (MILLER JP, et al.,2015). Por esta razão, uma infusão 15 mg IV de Cetamina administrado em bolus seguido imediatamente por uma infusão de $20 \mathrm{mg}$ de Cetamina administrada durante 1 hora proporcionou alívio da dor clinicamente significativo e sustentado, sem efeitos colaterais ou eventos adversos significativos que mudasse o manejo ou a intervenção necessária (AHERN TL, et al.,2015).

Portanto, a rápida redução da dor pelo uso de Cetamina em baixas doses é uma vantagem em comparação com opioides, como a morfina, para o tratamento da dor aguda no pronto-socorro. No entanto, a incapacidade de manter esse grau de alívio da dor durante o curso normal de uma internação no pronto-socorro pode exigir doses mais altas de Cetamina em baixa dose infundidas por um período mais longo ou 0 uso de medicamentos adjuvantes, onde analgesia prolongada é necessária (AHERN TL, et al.,2015).

\section{Não Farmacológicos}

Por fim, na emergência o tratamento não farmacológico da dor deve acima de tudo evitar mais danos e não deve demorar a resolução de lesões que coloquem em risco a vida do paciente (HÄSKE D, et al., 2017). Dessa forma, é encorajado que as intervenções não farmacológicas sejam aplicadas como adjuvantes ao tratamento farmacológico, a exemplo no período pós-operatório. No entanto, em situações de dor aguda, como nos departamentos de emergência dificilmente será possível associar tais práticas, devido à necessidade de estrutura, planejamento, bem como desenvolvimento e refinamento de determinadas técnicas que necessitam de discussão prévia com o paciente e seus familiares (MANWORREN R, 2015). 


\section{CONSIDERAÇÕES FINAIS}

Diante da revisão apresentada temos que a dor aguda do adulto não apenas é tema de grande relevância no departamento de emergência, mas também se mostra como alvo de diversos estudos científicos acerca de seu manejo. Assim, entre as particularidades do manejo álgico devem ser considerados o tempo de chegada do paciente ao $\mathrm{DE}$, da via de acesso pretendida, da disponibilidade de medicações, do mecanismo de ação das drogas, ou ainda, da associação destas, na chamada terapia multimodal. Isto é, associação entre um opioide à cetamina, ou a um AINE, promovendo conforto ao indivíduo, com o uso de doses mais baixas dos opioides, e, consequentemente, menores efeitos adversos. A despeito de poucos estudos envolvendo a terapia multimodal, os dados promissores revisados nesse artigo constatam a necessidade de melhor investigação dessa terapêutica, com intuito de aprimorar o seu manejo.

\section{REFERÊNCIAS}

1. AHERN TL, et al. Low-Dose Ketamine Infusion for Emergency Department Patients with Severe Pain. Pain Medicine. 2015; 16: 1402-1409.

2. ANDOLFATTO G, et al. Prehospital Analgesia with Intranasal Ketamine (PAIN-K): A Randomized Double-Blind Trial in Adults. Pain Management and Sedation. 2019; 74(2): 241-250.

3. BEAUDOIN FL, et al. Low-dose ketamine improves pain relief in patients receiving intravenous opioids for acute pain in the emergency department: results of a randomized, double-blind, clinical trial. Acad Emerg Med. 2014.

4. BERTONCELLO KCG, et al. Dor Aguda na Emergência: Avaliação e Controle com o Instrumento de MacCaffery e Beebe. Journal of Health Sciences. 2017;18(4).

5. BEVERLY A, et al. Essential elements of multimodal analgesia in enhanced recovery after surgery (ERAS) guidelines. Anesthesiology clinics. 2017; 35(2): e115-e143.

6. BLANCHER M, et al. Intranasal sufentanil versus intravenous morphine for acute severe trauma pain: A double-blind randomized non-inferiority study. PLoS Med. 2019.

7. BOWERS KJ, et al. Ketamine as an Adjunct to Opioids for Acute Pain in the Emergency Department: A Randomized Controlled Trial. Acad Emerg Med. 2017.

8. CHOU R, et al. Management of Postoperative Pain: A Clinical Practice Guideline from the American Pain Society, the American Society of Regional Anesthesia and Pain Medicine, and the American Society of Anesthesiologists' Committee on Regional Anesthesia, Executive Committee, and Administrative Council. J Pain. 2016.

9. CLATTENBURG EJ, et al. Intravenous Lidocaine Provides Similar Analgesia to Intravenous Morphine for Undifferentiated Severe Pain in the Emergency Department: A Pilot, Unblinded Randomized Controlled Trial. Pain Med. 2019.

10. KARLOW N, et al. A Systematic Review and Meta-analysis of Ketamine as an Alternative to Opioids for Acute Pain in the Emergency Department. Acad Emerg Med. 2018.

11. HAMRICK KL, et al. Multimodal analgesia and opioid use in critically ill trauma patients. Journal of the American College of Surgeons. 2019: 228(5): 769-775.

12. HÄSKE D, et alt. Analgesia in Patients with Trauma in Emergency Medicine. Dtsch Arztebl Int. 2017.

13. LEMOEL F, et al. Intranasal sufentanil given in the emergency department triage zone for severe acute traumatic pain: a randomized double-blind controlled trial. Intern Emerg Med. 2019.

14. MAJIDINEJAD S, et al. Comparison of Intravenous Ketamine with Morphine in Pain Relief of Long Bones Fractures: a Double Blind Randomized Clinical Trial. Emerg (Tehran). 2014.

15. MANWORREN RC. Multimodal pain management and the future of a personalized medicine approach to pain. AORN J. 2015.

16. $\mathrm{MAO} \mathrm{Y}$, et al. Perioperative Dexmedetomidine Fails to Improve Postoperative Analgesic Consumption and Postoperative Recovery in Patients Undergoing Lateral Thoracotomy for Thoracic Esophageal Cancer: A Randomized, Double-Blind, Placebo-Controlled Trial. Pain Research and Management. 2020.

17. MILLER JP, et al. Low-dose ketamine vs morphine for acute pain in the ED: a randomized controlled trial. American Journal of Emergency Medicine. 2015; 33(3): 402-408.

18. MOTOV S, et al. Intravenous Subdissociative-Dose Ketamine Versus Morphine for Analgesia in the Emergency Department: A Randomized Controlled Trial. Ann Emerg Med. 2015.

19. NOSANOV LB, et al. Pain Management in Burn Patients. Current Trauma Reports. 2020; 6: 161-173.

20. ROPPER AH, Nonnarcotic Methods of Pain Management. New English Journal of Medicine. 2019; 380(25): 24402448.

21. SIN B, et al. Intranasal Sufentanil Versus Intravenous Morphine for Acute Pain in the Emergency Department: A Randomized Pilot Trial. J Emerg Med. 2019.

22. VOLKOW ND, et al. Prevention and Treatment of Opioid Misuse and Addiction: A Review. JAMA Psychiatry. 2019. 